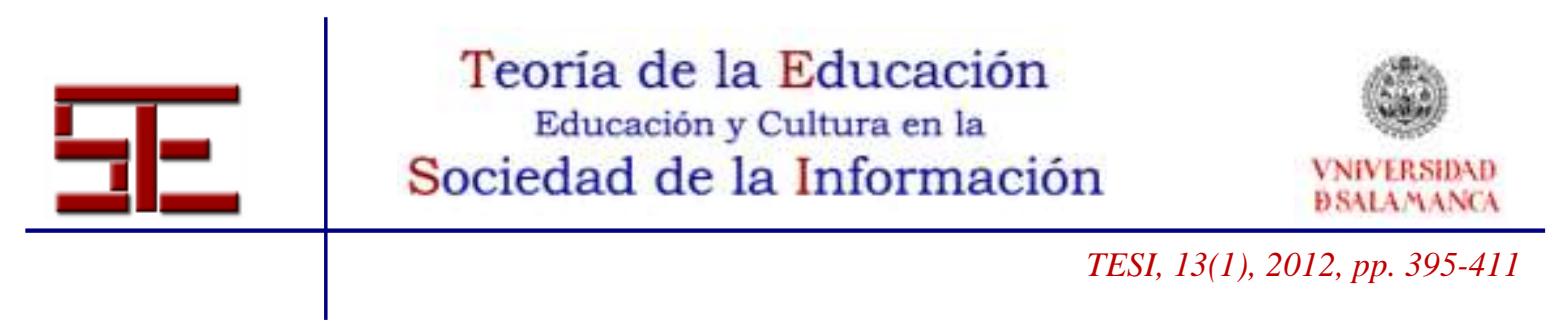

\title{
NUEVOS DESARROLLOS EN EL CAMPUS VIRTUAL UCM: ESTUDIO EXPLORATORIO SOBRE LAS PLATAFORMAS E-LEARNING EN LOS ESTUDIOS DE COMUNICACIÓN AUDIOVISUAL Y PUBLICIDAD
}

Resumen: La investigación en la que se basa este artículo tiene como objetivo principal el estudio de las capacidades de la actual herramienta del Campus Virtual de la UCM, WebCT 4.0, frente a las que presenta Moodle, el nuevo entorno de trabajo de dicho Campus, realizando un análisis en profundidad de la accesibilidad y usabilidad, de las herramientas de comunicación y de evaluación integral, así como de las funcionalidades multimedia.

La propuesta metodológica incluye técnicas tanto cualitativas como cuantitativas, planteándose un análisis exploratorio y descriptivo de las dos plataformas informáticas -a lo largo de tres fases consecutivas- que permita, en primer lugar, un estudio en profundidad de las herramientas utilizadas por el profesorado; en segundo lugar, la realización de un cuestionario orientado a conocer las valoraciones de los discentes acerca de la usabilidad, accesibilidad, así como de las herramientas de evaluación, comunicación y de gestión de contenidos; y, por último, la puesta en marcha de un experimento en el aula con los alumnos, simulando un contexto real de clase.

La usabilidad, la accesibilidad y las posibilidades de comunicación son más dinámicas en Moodle que en WebCT. Además, los alumnos demandan mayor participación mediante el uso de las redes sociales mayoritariamente extendidas.

Palabras clave: Entorno virtual; Tele-enseñanza; Campus Virtual; Nuevas Tecnologías; Moodle; Enseñanza superior; EEES.

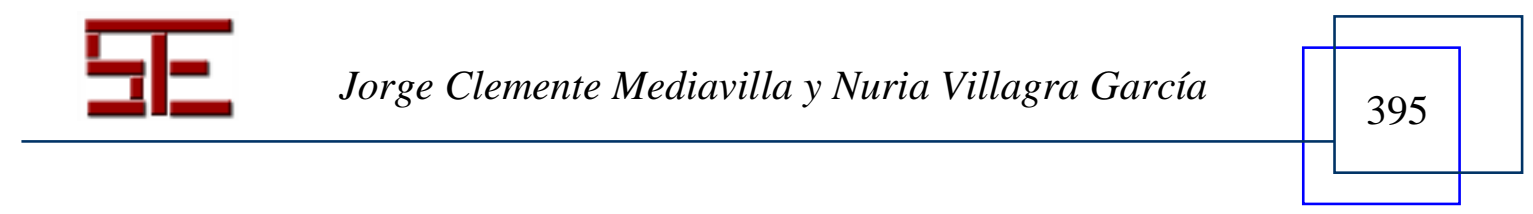




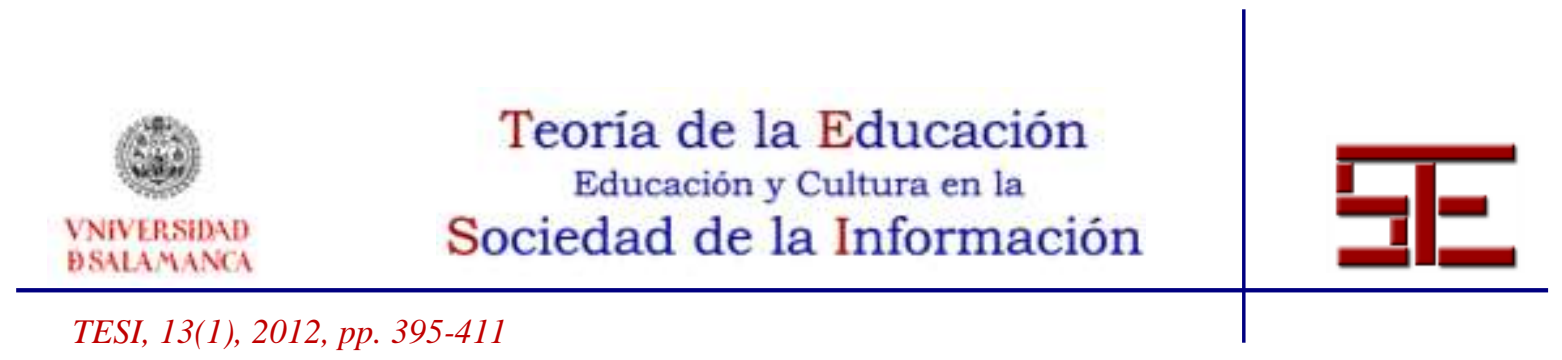

NEW DEVELOPMENTS IN THE VIRTUAL CAMPUS OF THE COMPLUTENSE UNIVERSITY: AN EXPLORATORY RESEARCH ON ELEARNING PLATFORMS IN THE STUDIES OF AUDIOVISUAL COMMUNICATION AND ADVERTISING

\begin{abstract}
This paper analyzes the capabilities of the present tool UCM Virtual Campus, WebCT 4.0, versus Moodle, the new working environment of the Campus. We examine accessibility and usability, communication tools and comprehensive assessment, as well as multimedia functionality.

The proposed methodology includes both qualitative and quantitative techniques. Over three consecutive phases this study analyzes the professor's experience using these tools. We also developed a questionnaire that was completed by the students in order to evaluate these e-learning platforms. Finally, the third phase of the research consisted of an experiment conducted in the classroom with the students simulating a real class context.
\end{abstract}

The usability, accessibility and the communication opportunities are more dynamic in WebCT than in Moodle. In addition, students demand a more participatory role by supplementing the use of social networks.

Keywords: Virtual platform; E-learning; Virtual Campus; New Technologies; Moodle; High Education; EHEA.

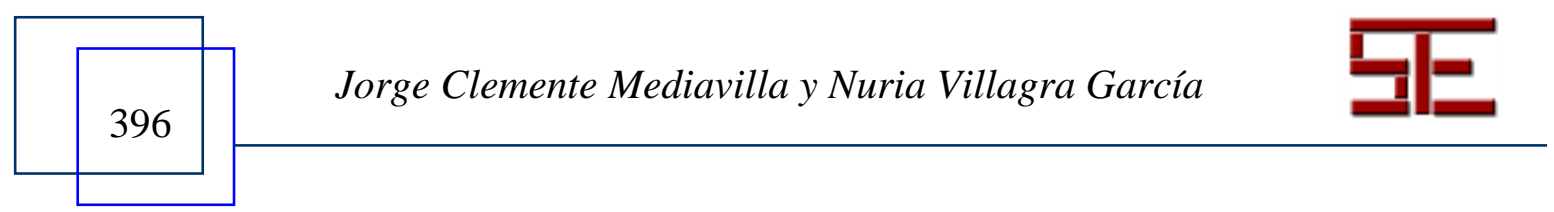




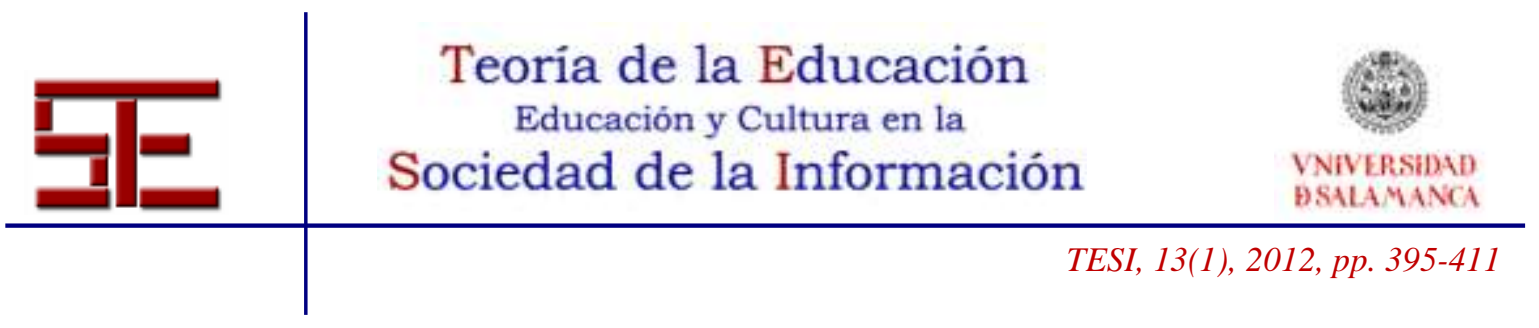

\section{NUEVOS DESARROLLOS EN EL CAMPUS VIRTUAL UCM: ESTUDIO EXPLORATORIO SOBRE LAS PLATAFORMAS E-LEARNING EN LOS ESTUDIOS DE COMUNICACIÓN AUDIOVISUAL Y PUBLICIDAD}

Fecha de recepción: 10/07/2011; fecha de aceptación: 29/01/2012; fecha de publicación: 05/03/2012

Jorge Clemente Mediavilla

jclemen@ccinf.ucm.es

Universidad Complutense de Madrid

Nuria Villagra García

nuriavillagra@ccinf.ucm.es

Universidad Complutense de Madrid

\section{1.- INTRODUCCIÓN}

\section{1.- Fundamentación}

La integración de las tecnologías de la información y la comunicación (TIC) ofrece unas enormes posibilidades en los procesos educativos (Clares, 2000; Cortina-Pérez, 2008; Garrison \& Anderson, 2005; Salmerón, Rodríguez \& Gutiérrez, 2010), tanto en la formación presencial, como semipresencial o a distancia. En concreto, está provocando cambios en la naturaleza y procesos de enseñanza, en la relación entre alumnos y profesores y en las modalidades educativas ofertadas por las universidades. Todo lo anterior coincide en el tiempo con el proceso de implantación de un nuevo paradigma de formación que propone el Espacio Europeo de Educación Superior (EEES), basado en la adquisición de competencias y en la innovación educativa. En este nuevo modelo el alumno debe implicarse y participar de un modo activo en el proceso de aprendizaje, de ahí que la incorporación de herramientas e-learning favorezca la consecución de este objetivo al mismo tiempo que integran las TIC en el proceso formativo. Como señala Van Dusen (1997), la introducción de la tecnología en el proceso educativo incorpora indudables ventajas ya que impulsa el aprendizaje colaborativo y cooperativo, el desarrollo de grupos heterogéneos, la solución de problemas y el pensamiento reflexivo, que una clase presencial por sí sola no puede facilitar. Todo lo anterior ha puesto de manifiesto en los últimos años retos interesantes que afectan a alumnos, profesores y a

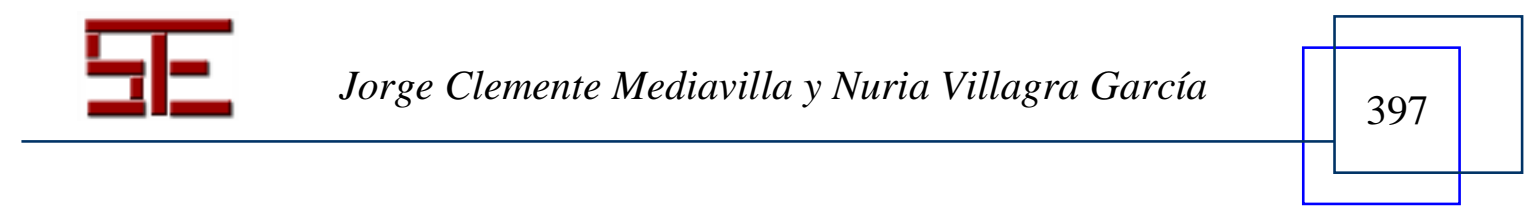




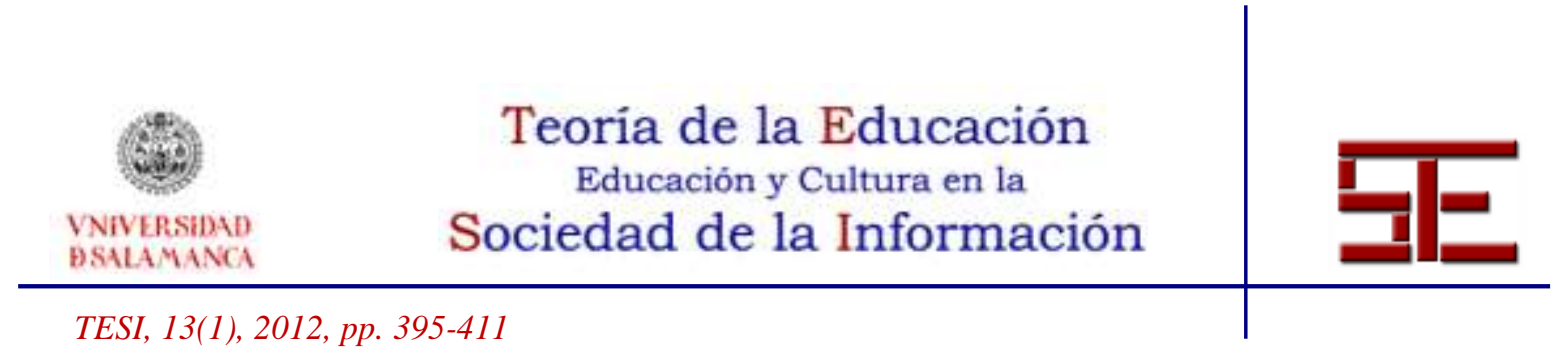

la propia forma de entender la educación en el contexto de las universidades presenciales.

En este artículo se trata de dirigir la atención hacia el futuro inmediato, que tiene que ver con la implantación del Espacio Europeo de Educación Superior (EEES) y los cambios que dicha instauración está suponiendo para la universidad española en general, y para la Universidad Complutense en particular. Derivado de lo anterior, podemos afirmar que el Campus Virtual es una plataforma docente que se está utilizando cada vez más en las distintas universidades de nuestro país, al igual que en el resto del mundo, y a la cual nos vamos a ver abocados sin duda en un plazo muy corto de tiempo, una vez quede implantado definitivamente el EEES.

Por otro lado, resulta evidente que el alumno universitario demanda cada día más el uso de las nuevas tecnologías en el proceso de aprendizaje. Por este motivo, la satisfacción del estudiante en relación con la calidad de la enseñanza está cada vez más afectada por el grado de desarrollo de los sistemas de Campus Virtual. En consecuencia, el diseño y la usabilidad de las plataformas deben incidir en las necesidades, requisitos y limitaciones con las que se puede encontrar el usuario final. Es más, las plataformas elearning deben adaptarse tanto a las características de la docencia como a las de los usuarios. Por tanto, el impacto de la usabilidad se convierte en un punto de referencia fundamental en el proceso de convergencia hacia el EEES. El Campus Virtual no es sólo una plataforma de aprendizaje, sino una experiencia de comunicación entre alumnos y profesores y, por extensión, entre los propios docentes que desarrollan materias complementarias. La necesidad de integrar todos estos conceptos en el Campus Virtual debe convertirse en un punto fundamental a la hora de desarrollar sistemas y procesos de programación docente tanto desde un punto de vista intrínseco, es decir, entre materias comunes en un Grado, como desde un punto de vista extrínseco, entre materias interdisciplinares.

\section{2.- Campus Virtual: concepto y tipos de plataformas tecnológicas}

Van Dusen (1997) define el Campus Virtual como una metáfora de la enseñanza, el aprendizaje y la investigación creado por la convergencia de las nuevas tecnologías de la comunicación. El Campus Virtual es una herramienta de e-learning que permite desarrollar los recursos telemáticos característicos de Internet (páginas web, correo, chat, foro, videoconferencia, etc.) dentro de un único espacio al que pueden acceder profesores y alumnos. Dicho espacio es gestionado por el profesor y se adapta a las

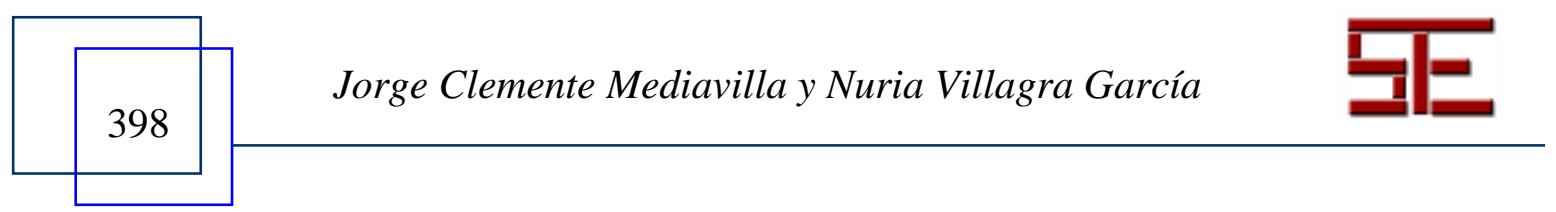




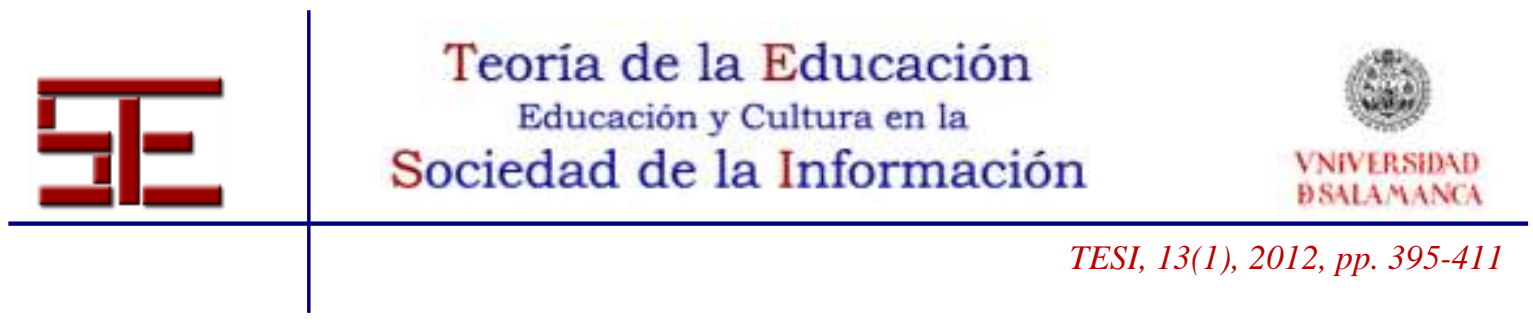

estrategias docentes que éste encuentre más adecuadas, en función de variables tales como la metodología docente empleada, el área de conocimiento o el nivel académico. Como señala Area (2001), este espacio educativo virtual puede servir para el desarrollo de dos funciones pedagógicas principales:

- Campus Virtual como apoyo a la docencia presencial. En este caso su principal función es la de facilitar la integración y uso de los recursos de Internet (archivos multimedia, documentos de apuntes o apoyo, chats, correo electrónico, etc.) en las clases convencionales.

- Campus Virtual como escenario para la educación a distancia. Esta segunda opción permite a los alumnos cursar estudios universitarios aunque no puedan asistir al aula; por tanto, no es complemento a las clases presenciales sino, que es la referencia básica del proceso formativo.

Las características de este entorno de enseñanza virtual deben ser definidas por cada universidad de acuerdo a las necesidades educativas y funcionales que requiera dicha institución. En este sentido, en los últimos años se han desarrollado numerosas plataformas tecnológicas que las universidades escogen y adaptan a sus propias necesidades. El proceso de selección puede tener en cuenta muy diversos aspectos (Llorente, 2007), tales como las necesidades formativas que se quieran cubrir, el modo de adquisición y distribución de dicha plataforma en el mercado o la filosofía pedagógica que ha inspirado su diseño. No es objeto de este trabajo realizar un estudio pormenorizado y comparativo de cada una de las plataformas, ya que en este sentido existen trabajos anteriores como los realizados por Belanger y Jordan (2000), Novitzki (2000) o Llorente (2007). Nuestra investigación parte de la distinción entre plataformas de software comercial y de software libre, analizando un ejemplo concreto de cada una de ellas. De forma específica esta investigación centró su atención en la Universidad Complutense donde se da una situación poco frecuente, ya que conviven tres entornos virtuales de enseñanza: Moodle, WebCT y Sakai, siendo las más empleadas las dos primeras. Por tanto, cada profesor en función de sus preferencias emplea mayoritariamente en su asignatura el entorno Moodle o WebCT.

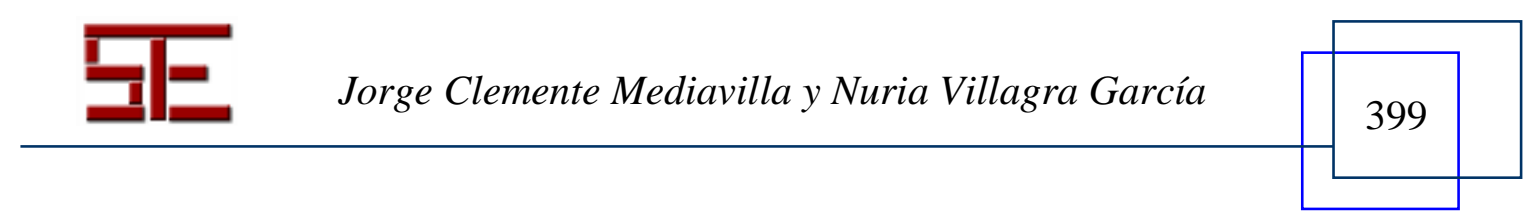




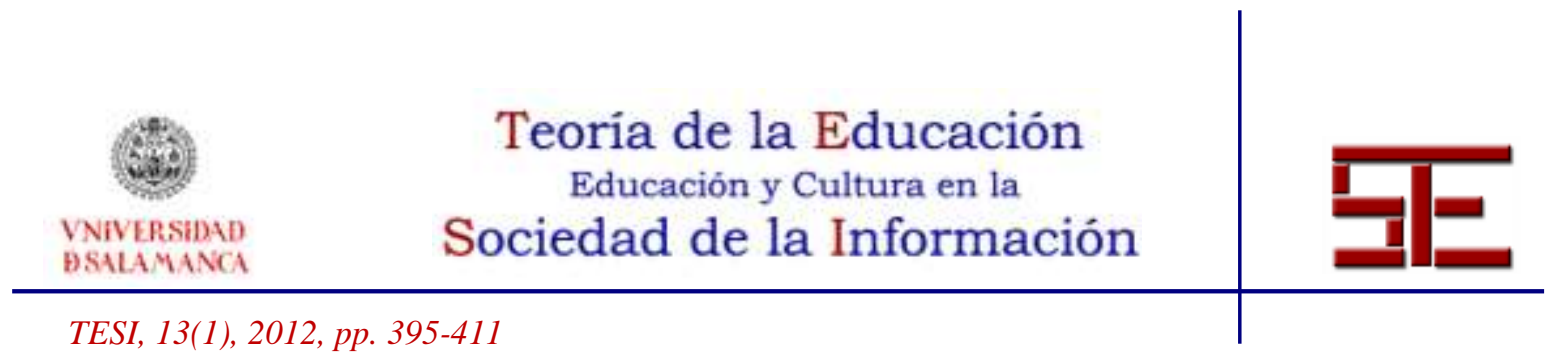

\section{2.- OBJETIVOS Y METODOLOGÍA}

\section{1.- Objetivos}

El principal objetivo de la investigación se relaciona con el avance de la herramienta Campus Virtual entre la comunidad universitaria, proponiendo mejoras basadas en un análisis de usabilidad y de las herramientas de gestión existentes y del desarrollo de nuevas funcionalidades, con el fin de fomentar la puesta en común de conocimientos, que lleve a un aumento de la productividad educativa y científica, configuradas como dos pilares básicos del modelo universitario.

Desde una perspectiva más práctica, se pretende estudiar las capacidades de la actual herramienta del Campus Virtual de la UCM, WebCT 4.0, frente a las que presenta Moodle, el nuevo entorno de trabajo de dicho Campus. Aunque a corto plazo se mantendrán las herramientas de virtualización bajo plataforma WebCT, los planes de desarrollo del Campus pasan por una migración completa a otros entornos de los que Moodle, por su amplísima difusión y comunidad de usuarios global, se perfila como el más conveniente. La presencia simultánea de dos entornos e-learning en una misma institución permitía analizar de forma comparativa los principales aspectos de ambas plataformas, con el fin de proponer mejoras en el diseño de la plataforma Moodle. Desde una perspectiva más específica y respecto a ambas plataformas, se programó el estudio en profundidad de las aplicaciones más utilizadas por docentes y alumnos, tales como herramientas de comunicación y de evaluación integral, y además se buscaba obtener una visión clara de las funcionalidades multimedia (tan importantes en las titulaciones estudiadas), así como de la accesibilidad y la usabilidad.

En cuanto a objetivos específicos, se plantean los siguientes:

- Análisis comparativo de las herramientas de gestión de contenidos y espacios en WebCT y Moodle, desde el punto de vista del profesor.

- Análisis de las herramientas de comunicación de WebCT y Moodle, desde el punto de vista del profesor y de los alumnos.

- Estudio de estrategias y herramientas de evaluación integral bajo WebCT y Moodle, desde el punto de vista del profesor y de los alumnos.

- Análisis de accesibilidad y usabilidad en WebCT/Moodle y diseño de reglas para la creación de espacios accesibles, desde el punto de vista del profesor y de los alumnos.

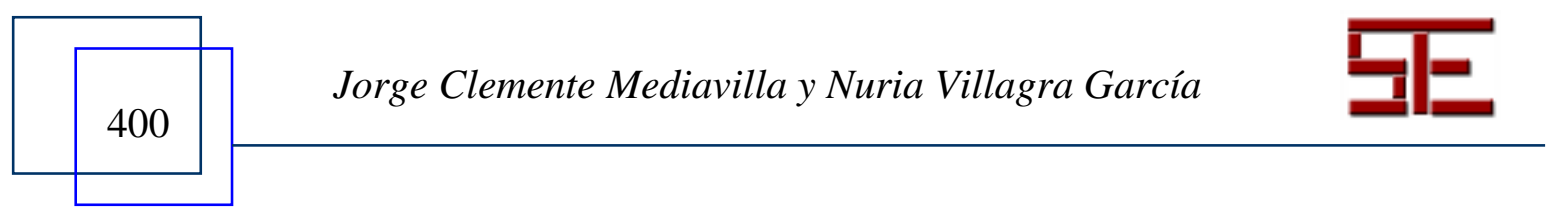




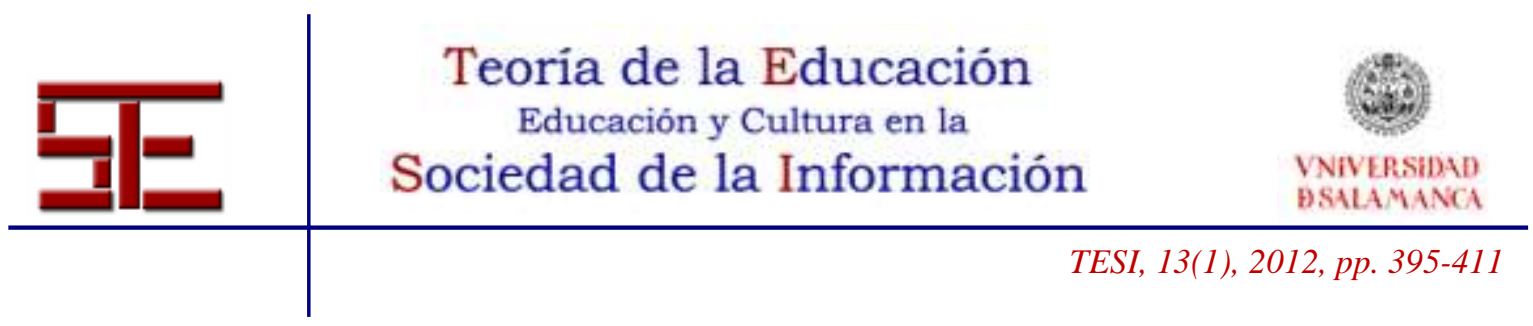

\section{2.- Metodología}

Tratando de responder a los objetivos y teniendo en cuenta trabajos anteriores realizados en nuestro país (Area, 2001; Cabero, 2002; Llorente, 2007; Pérez, 2008; Salmerón, Rodríguez \& Gutiérrez, 2010) se definió una metodología que combina técnicas cualitativas y cuantitativas llevadas a cabo en tres fases consecutivas durante el curso 2009/10:

\subsection{1.- Primera fase: Experimento profesores}

En esta primera fase se definió un experimento consistente en el análisis y la creación de espacios gemelos en Moodle y WebCT. Para ello fueron seleccionados dos profesores de la Facultad de Ciencias de la Información con un conocimiento avanzado de plataformas e-learning, que impartían asignaturas relacionadas con nuevas tecnologías, para que realizaran un análisis descriptivo y comparativo de ambas plataformas. Atendiendo a un planteamiento eminentemente cualitativo, a cada uno de ellos se le asignó una de las plataformas de estudio y se les pidió que elaboraran un espacio de trabajo virtual con características similares que les permitiera desarrollar distintas actividades, así como comparar la usabilidad y funcionalidad de cada uno de estos entornos e-learning. Esta primera fase permitió obtener datos por observación directa y basados en la propia experiencia de los docentes acerca de las principales ventajas, limitaciones o diferencias de operatividad existentes entre cada una de las plataformas analizadas. También permitió observar tipos de contenidos y actividades soportadas por ambas plataformas. Es decir, se centró en analizar el Campus Virtual como herramienta de apoyo a la docencia desde el punto de vista del profesor.

\subsection{2.- Segunda fase: Encuesta a alumnos}

Para llevar a cabo el estudio se seleccionó una muestra de 246 alumnos de segundo ciclo de los estudios de Comunicación Audiovisual y de Publicidad y Relaciones Públicas, de la Facultad de Ciencias de la Información. El cuestionario constaba de un total de veintiséis preguntas de distintos tipos: de respuesta cerrada, de valoración por escalas de Likert de cinco puntos y preguntas abiertas. El acceso al cuestionario se realizaba a través de un enlace ubicado en el Campus Virtual, del cual se informó a los alumnos durante las clases y por medio del correo electrónico.

Los bloques de contenido en los que se estructuraba el cuestionario eran los siguientes:

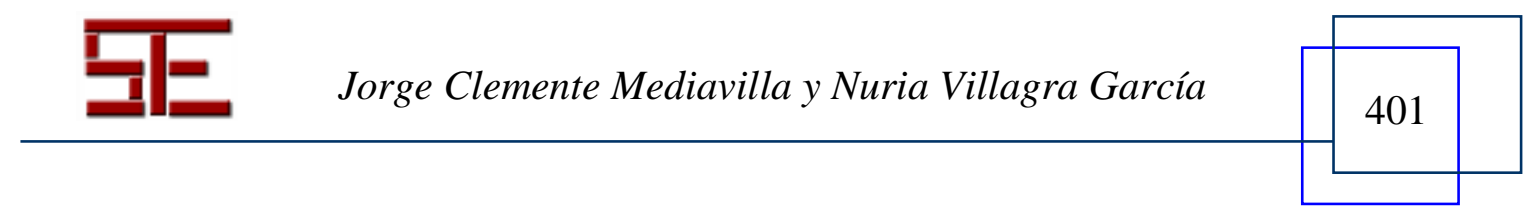




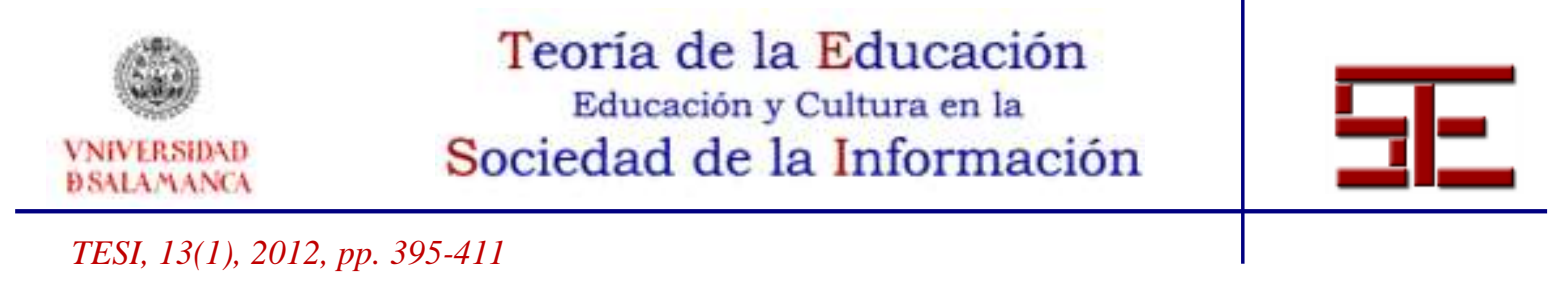

Bloque 1: Preguntas sobre el perfil sociodemográfico de la muestra y contexto de acceso al Campus Virtual

En este bloque se preguntaba a los entrevistados por variables como el sexo o la edad y además se incluían una serie de preguntas cerradas acerca de la forma de acceso: plataforma electrónica empleada (PC, MAC, móvil, etc.), sistema operativo y navegador. También incluía preguntas acerca de la frecuencia, tiempo y lugar de acceso, principales utilidades empleadas, consulta del Campus Virtual durante las clases, número de asignaturas virtualizadas dentro de su curso y plataforma empleada (Campus Virtual, web del profesor, etc.).

Bloque 2: Valoración de Moodle como entorno e-learning

Contenía una serie de preguntas en las que los alumnos a través de escalas de Likert y preguntas abiertas valoraban los siguientes aspectos:

- Usabilidad y accesibilidad de Moodle como espacio docente.

- Herramientas de contenido (archivos, carpetas, documentos audiovisuales, etc.).

- Herramientas de comunicación entre profesor y alumnos (chat, e-mail, foro, etc.).

- Herramientas de evaluación.

Bloque 3: Valoración de WebCT como entorno e-learning

Contenía las mismas preguntas que el bloque 3 pero centradas en el entorno WebCT. Es interesante señalar que dado que ambas plataformas son utilizadas indistintamente por los profesores, durante el curso académico los discentes estaban empleándolas simultáneamente y podían valorar su experiencia en las distintas asignaturas que cursaban. Por tanto, en esta segunda fase se analizaban las valoraciones, percepciones y experiencias de los alumnos para cada una de las plataformas de estudio.

\subsection{3.- Tercera fase: Experimento en el aula}

Con el fin de observar directamente el uso que los alumnos realizan del Campus Virtual y comprobar su empleo como herramienta didáctica durante el desarrollo de las clases

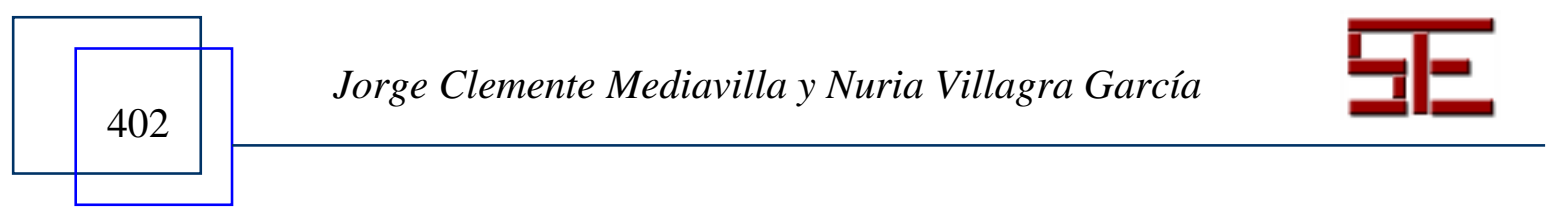




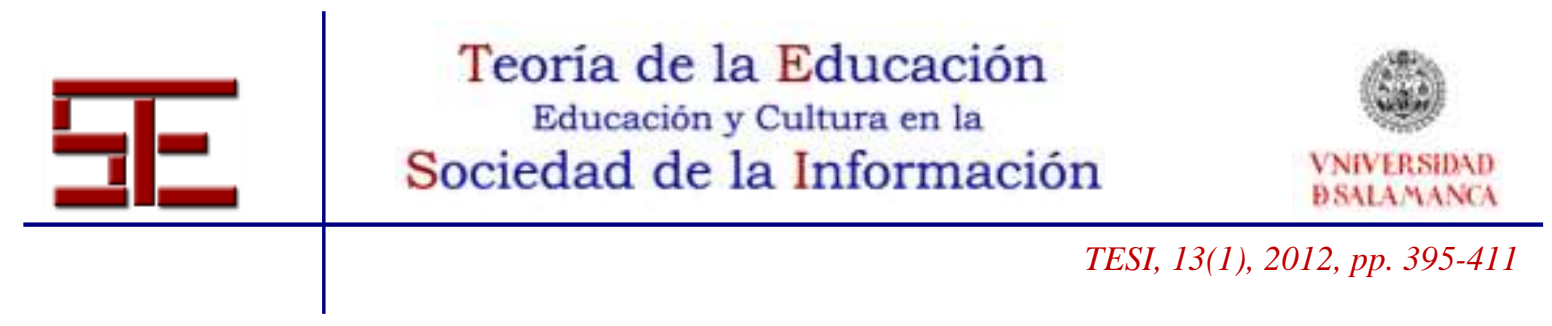

presenciales, se definió un experimento (Campbell \& Stanley, 1995) que tuvo lugar en un aula de informática en la que cada alumno dispuso de un ordenador para trabajar. Se seleccionaron dos grupos reducidos de alumnos (participaron dieciocho individuos en cada caso). En dicho experimento se simulaba una actividad desarrollada dentro del horario lectivo, y posteriormente se facilitaba a los alumnos un cuestionario para que valoraran la facilidad en la descarga de contenidos y acceso a documentos en distintos formatos en la plataforma Moodle o WebCT. Este experimento permitió utilizar el Campus Virtual en el contexto del aula y observar directamente el uso y experiencia del mismo por parte de los alumnos.

A continuación se reflejan los principales resultados obtenidos en cada una de las fases.

\section{3.- RESULTADOS}

\section{1.- Resultados análisis plataformas informáticas}

En primer lugar, es reseñable que en ambas plataformas la vinculación de un usuario con capacidades de edición ha de realizarse de forma externa a los espacios virtualizados, ya que desde las herramientas de gestión de usuarios incorporadas a los mismos pueden únicamente cambiarse algunos privilegios de menor importancia o controlar el acceso de los mismos a algunos contenidos del espacio virtualizado.

Además, las herramientas de creación y adquisición de contenidos son similares en WebCT y Moodle y presentan parecida funcionalidad, aunque la segunda plataforma admite un abanico más amplio de posibles contenidos a incorporar, con una mayor compatibilidad con aplicaciones y formatos externos. Es especialmente destacable la posibilidad de inclusión de contenidos con capacidades interactivas o de funcionamiento en línea que admite Moodle.

En cuanto a la edición y gestión de contenidos creados, existen mayores posibilidades en la plataforma Moodle. Los cambios sobre los contenidos realizados en dicha plataforma son inmediatamente visibles por los usuarios del espacio virtual, lo que permite una operación de mantenimiento o actualización de contenido siempre en línea y más natural que en WebCT, que necesita una operación de actualización para validar y hacer visibles los cambios introducidos. Además, es destacable que Moodle admite un mayor control sobre parámetros de acceso, visualización o descarga de contenidos que los que presenta WebCT, incluyendo filtros de acceso condicional a los mismos que

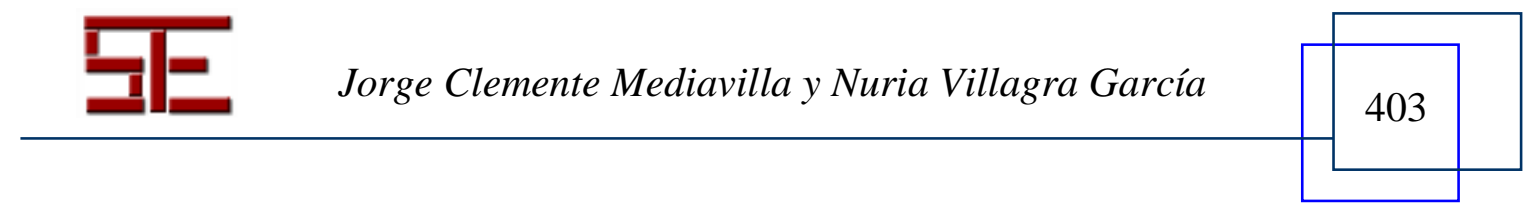




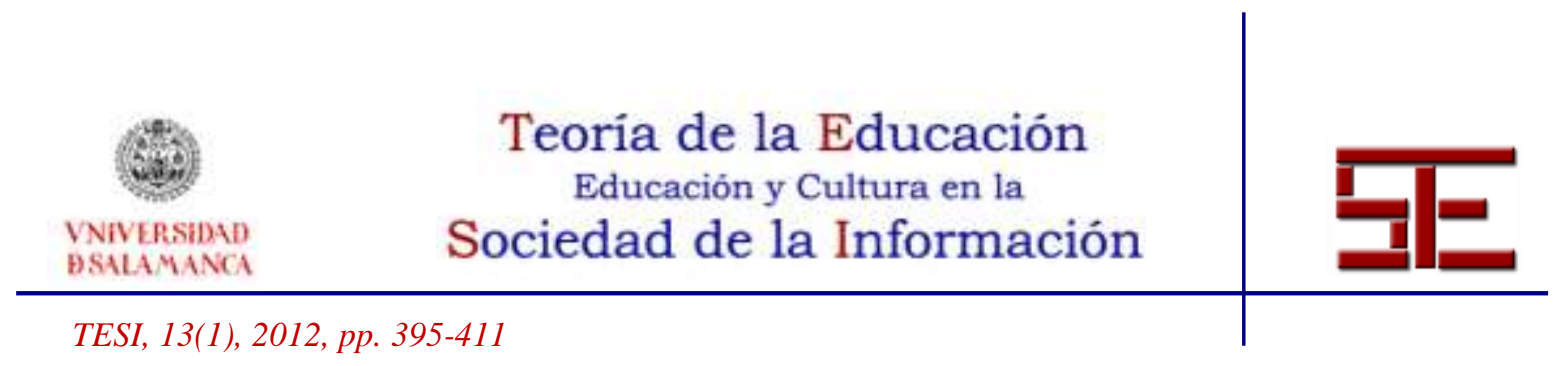

pueden ser configurados de acuerdo con parámetros temporales o asociados a los perfiles de los usuarios vinculados al espacio virtual.

Y en lo referente a la generación de estructuras de curso o de módulos de contenidos, WebCT presenta un único modelo de arquitectura mientras que Moodle permite elegir entre varias posibilidades básicas para la estructura general del curso o espacio, con el fin de adecuar mejor el desarrollo del mismo al modelo de enseñanza empleado o al escalón formativo en el que se encuadra el espacio.

\section{2.- Resultados encuesta online}

\subsection{1.- Resultados respecto del contexto de acceso al Campus Virtual}

Cuando se preguntó a los alumnos qué plataforma utilizaban más habitualmente para acceder al Campus Virtual, la mayoría señaló el PC $(85,94 \%)$ seguido a bastante distancia de MAC $(10,94 \%)$. Otras plataformas tales como el móvil son todavía muy minoritarias, y sólo el 1,56\% la eligió como plataforma habitual de acceso. Además, coincidiendo con los resultados anteriores, el sistema operativo que los alumnos utilizaban de forma más habitual para acceder a los contenidos del Campus fue Windows (84,38\%), seguido de MAC OS (10,94\%) y Linux (4,69\%).

En lo que se refiere al navegador, destacaron dos con porcentajes muy similares: en primer lugar Mozilla que es empleado por el 46,88\% de los encuestados, seguido muy de cerca por Explorer con un 42,19\%, y a bastante distancia de los anteriores se situaron Safari $(9,38 \%)$ y Opera $(1,56 \%)$.

Por otro lado, los alumnos consultaron regularmente los contenidos del Campus Virtual, de modo que un 40,63\% afirmó acceder varias veces por semana, seguido de una vez al día $(18,75 \%)$ y una vez por semana $(18,75 \%)$. El 14,06\% indicó que accedió varias veces al mes, el 6,25\% aseguró acceder más de una vez al día y, finalmente, sólo un $1,56 \%$ señaló que accedió una vez al mes o menos.

Aunque la frecuencia de acceso es elevada el tiempo de estancia no es muy alto, ya que la mayoría $(65,08 \%)$ permaneció menos de 15 minutos, seguido de un $31,75 \%$ que consultó los contenidos por un tiempo que osciló entre los 15 minutos y una hora. Las opciones "entre una y dos horas" y "más de dos horas" se eligieron únicamente por un $1,56 \%$ en cada caso.

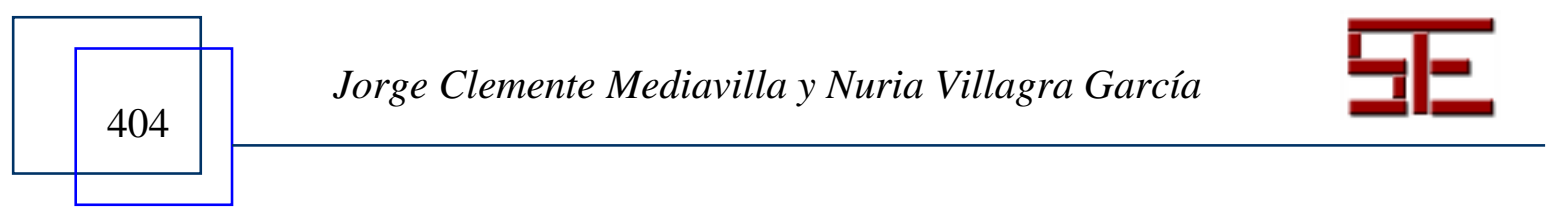




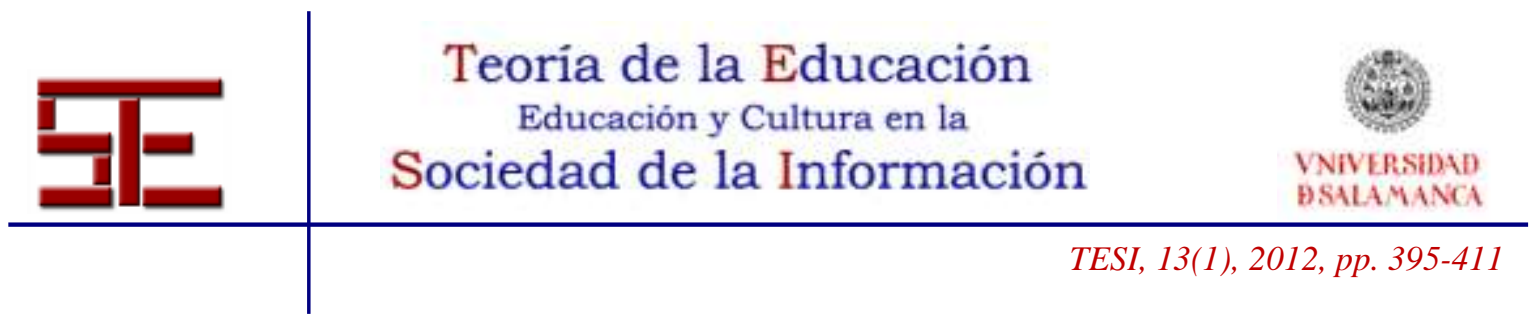

Respecto a la forma de acceso, los alumnos utilizaron distintas opciones, según el lugar en el que se encontraran. Las citadas más frecuentemente fueron: ordenador portátil $(81,25 \%)$, facultad $(56,25 \%)$ y ordenador de casa $(45,31 \%)$. Otras formas de acceso, aunque menos habituales, fueron: lugares públicos con Wi-Fi $(12,50 \%)$ y móvil $(3,13 \%)$.

Los alumnos consultaron el Campus Virtual tanto durante el desarrollo de las clases como fuera de las mismas; sin embargo, lo más habitual es que accedieran fuera de las clases $(96,88 \%)$ y sólo un $29,69 \%$ lo hizo además durante las clases; únicamente un $3,13 \%$ señala que accedió en el transcurso de la clase. Estos resultados ponen de manifiesto que, aunque la mayoría tuvo acceso al Campus Virtual fuera del aula, hay un porcentaje considerable que lo hizo durante el desarrollo de la docencia. Actualmente se observa que, aunque el porcentaje de alumnos que poseen un portátil es elevado $(81,25 \%)$, son pocos los que lo llevan a clase y lo utilizan en ese contexto para acceder a los contenidos.

En lo referente al uso del Campus Virtual por parte de los discentes, se observa que las opciones señaladas más frecuentemente fueron las referidas a contenidos y consulta de notas. Así, la descarga de contenidos del curso es lo que buscan la mayoría de los alumnos que acceden $(98,44 \%)$, mientras que un $85,94 \%$ también utiliza el Campus Virtual de forma intensiva para consultar las notas y un $82,81 \%$ para ver el programa de las asignaturas. Las herramientas de comunicación o trabajo en grupo son muy poco utilizadas y sólo el envío de correos electrónicos al profesor obtuvo un porcentaje elevado en este apartado $(60,94 \%)$. El resto de usos consiguieron resultados muy bajos: "envío de correos electrónicos a mis compañeros" (15,63\%), "trabajar en grupo" $(14,06 \%)$, "participar en foros" (14,06\%), "otros usos" -en este apartado los alumnos indicaron que realizaron cursos on line- $(6,25 \%)$ y "participación en chats" $(1,56 \%)$.

\subsection{2.- Resultados comparativa WebCT y Moodle.}

Respecto al análisis de las herramientas de gestión de contenidos (textuales, audiovisuales, etc.), en WebCT y Moodle, los resultados arrojan una buena valoración de ambas plataformas por parte de los alumnos encuestados, si bien existen diferencias: mientras que en la plataforma WebCT un 79,63\% señala que los materiales del curso (apuntes, carpetas de contenidos, programa, etc.) se encuentran fácilmente y están agrupados de forma lógica, en Moodle un 51,42\% señala estar muy de acuerdo o totalmente de acuerdo con esta afirmación, lo cual refleja que existe un número elevado

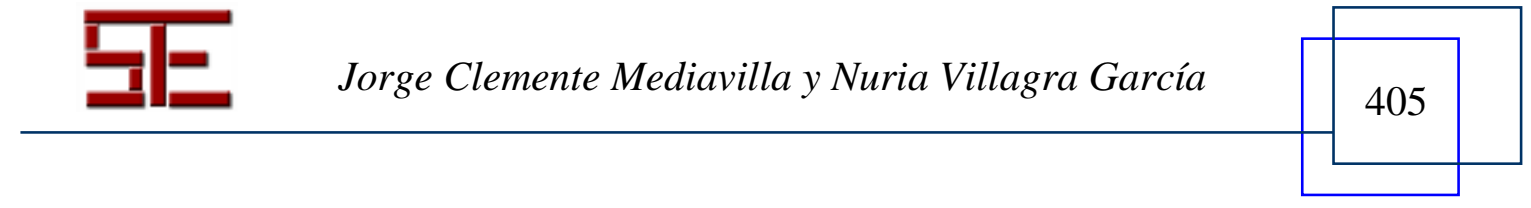




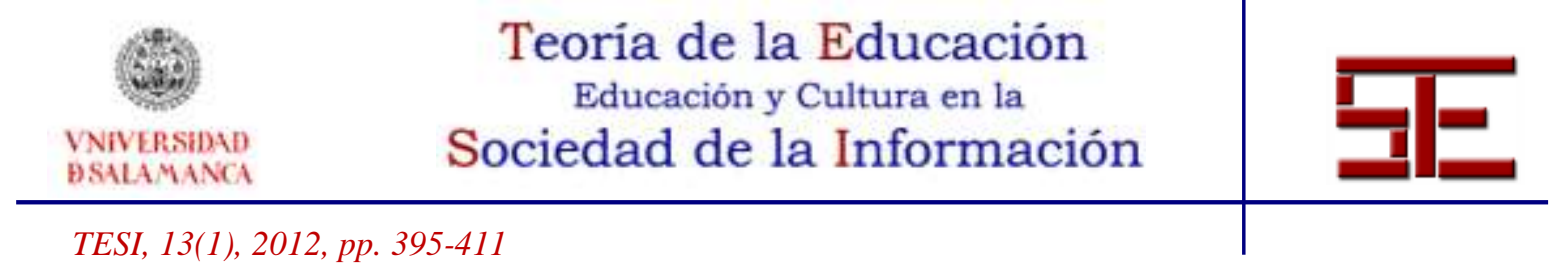

de alumnos que no se sienten plenamente satisfechos. De otra parte, en WebCT los alumnos consideran que la descarga de dichos archivos es fácil y rápida: en concreto un $61,10 \%$ valora muy bien dicho uso; en Moodle, los alumnos también afirman claramente que la descarga de dichos archivos es fácil y rápida, ya que un 55,99\% declara que las descargas son correctas. Cuando se les preguntó por la posibilidad de un espacio para subir y compartir archivos con los compañeros la mayoría era favorable, ya que en Web CT un 64,81\% valoró muy positivamente dicha opción, y en Moodle fue un $76,48 \%$, observándose entonces que en ambas plataformas existe una demanda por parte de los discentes para utilizar el Campus de una forma más participativa y activa.

Asimismo, se preguntaba al alumnado por el tipo de contenidos que había consultado o descargado durante el curso y cuál entendía que era su grado de utilidad. Los contenidos más consultados fueron el programa y los archivos de apuntes, presentaciones y otra documentación aportada por el profesor. Tanto en WebCT como en Moodle se obtuvieron puntuaciones muy elevadas a la hora de valorar su utilidad: en WebCT un $58,18 \%$ consideró que el programa es un contenido muy útil y un $56,36 \%$ opinó lo mismo acerca de los archivos y apuntes, mientras que en Moodle un 48,57\% consideró que el programa era un contenido muy útil y un 47,06\% pensó lo mismo acerca de los archivos y apuntes.

También se pedía a los alumnos que destacaran los aspectos que más valoraban de la plataforma y aquellos que entendían que debían mejorarse. En WebCT destacaron la gran capacidad de almacenamiento de contenidos $(36,84 \%)$ y la facilidad de búsqueda de contenidos $(21,05 \%)$, mientras que en Moodle valoraron positivamente la facilidad tanto de la visualización de las actualizaciones de contenidos como de la búsqueda de los contenidos vertidos por el profesor. En lo que se refiere a los aspectos que podrían mejorarse, en WebCT las opiniones más significativas son la "escasez de contenidos de la plataforma" (31,58\%) y la "escasez de contenidos por parte de los profesores" (31,58\%), mientras que en Moodle podemos resaltar su opinión sobre la escasez de contenidos que los profesores depositan en la plataforma.

En cuanto al análisis de las herramientas de comunicación de WebCT y Moodle, los resultados arrojaron la existencia de diferencias significativas entre ambas plataformas. En primer lugar, Moodle supone para el alumnado un interfaz comunicativo más atractivo y dinámico que WebCT e incorpora además una herramienta de comunicación adicional que es el Blog. Sin embargo, se observa en ambas plataformas que, independientemente de las herramientas que ofrecen, en la actualidad estas son

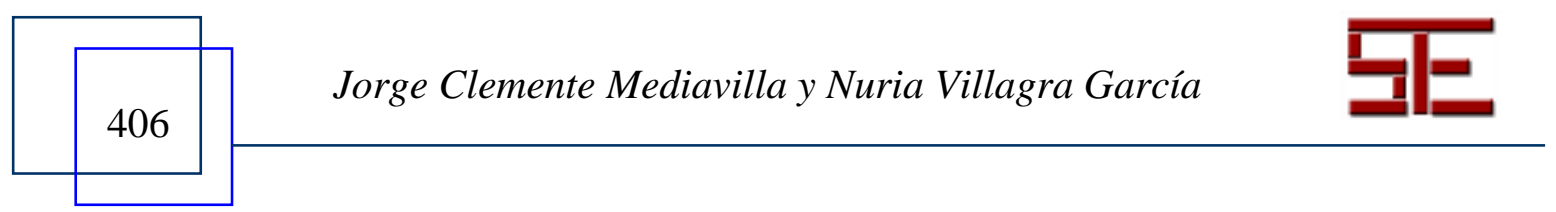




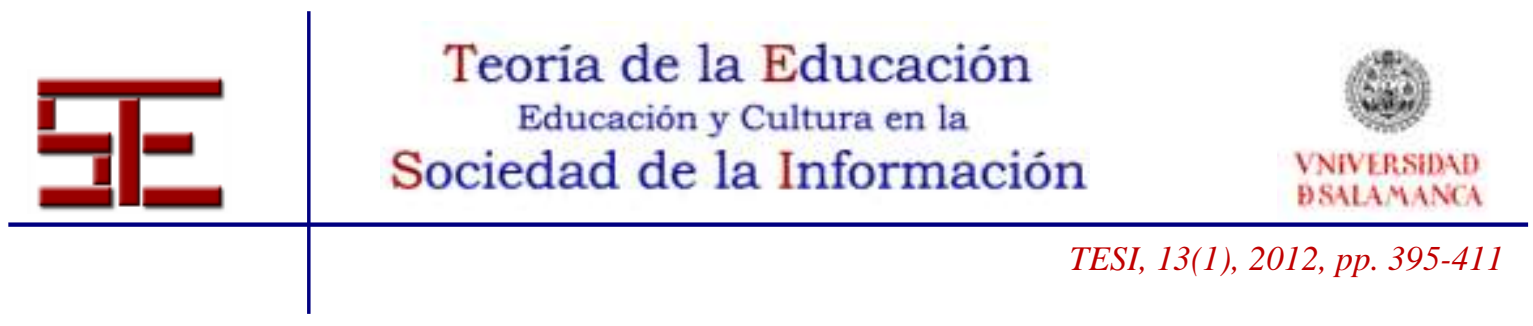

infrautilizadas por el profesorado: con excepción del correo, que es la más empleada, el $77,14 \%$ la ha usado en Moodle y el 83,33\% en WebCT. El resto de herramientas de comunicación tienen un uso prácticamente inexistente, como en el caso del Chat, el Blog, el Foro, etc. Incluso en el caso del Correo, que es la herramienta más utilizada, la frecuencia de acceso es muy baja: sólo un 5,71\% la utiliza más de una vez al día y un 8,57\% una vez al día (Moodle); en el caso de WebCT un 3,70\% más de una vez al día y un 5,56\% una vez al día. En lo referente a la valoración que los alumnos realizaron de las mismas, las mejor puntuadas coinciden con las más usadas: en el caso de Moodle son el Correo y el Foro, y en el caso de WebCT, Correo, Foro de Debate y Grupo de Trabajo.

Respecto al estudio de estrategias y herramientas de evaluación integral bajo WebCT y Moodle, hemos de decir que las herramientas de evaluación que ofrece la plataforma Moodle son bien distintas de las de WebCT, lo que ha dificultado la comparativa entre ambas plataformas. Los resultados obtenidos en Moodle indican que las herramientas de evaluación tienen una utilización escasa: la Autoevaluación es la que menos se ha usado $(85,71 \%)$, seguida por el Cuestionario $(65,71 \%)$, las Encuestas $(62,86 \%)$, las Tareas $(58,82 \%)$ y las Lecciones $(54,29 \%)$. En WebCT el empleo de herramientas de evaluación es muy limitado: la Autoevaluación es la que menos se ha usado $(85,71 \%)$, seguida por el Cuestionario (65,71\%), las Encuestas $(62,86 \%)$, las Tareas $(58,82 \%)$ y las Lecciones $(54,29 \%)$. Respecto a la valoración de estas herramientas, en el caso de Moodle la mejor puntuada es Lecciones (un 34\% de los encuestados la consideran útil o muy útil), mientras que en el caso de WebCT la mejor valorada es Mis Calificaciones, con un $83,64 \%$ favorable.

En lo referente al análisis de accesibilidad y usabilidad en WebCT/Moodle y el diseño de reglas para la creación de espacios accesibles, las valoraciones son positivas en ambas plataformas; sin embargo, en todas las dimensiones analizadas WebCT obtiene valoraciones más altas. Las afirmaciones sobre las que se pedía su grado de acuerdo eran las siguientes: "Accedo a cualquier contenido o herramienta con facilidad y sin tener que realizar más de tres pasos", "Desde el primer momento me pareció intuitivo y fácil de usar", "Las etiquetas que identifican las distintas actividades son adecuadas y permiten localizar lo que se busca en el menú de forma rápida", "El menú con las principales opciones de navegación está presente en todo momento y es fácil acceder a los distintos contenidos", "A veces cuando he navegado por distintas pantallas dentro de las páginas, me siento perdido y no es fácil volver a lo que estaba consultando anteriormente" y "El diseño de la página es atractivo y funcional para un uso docente".

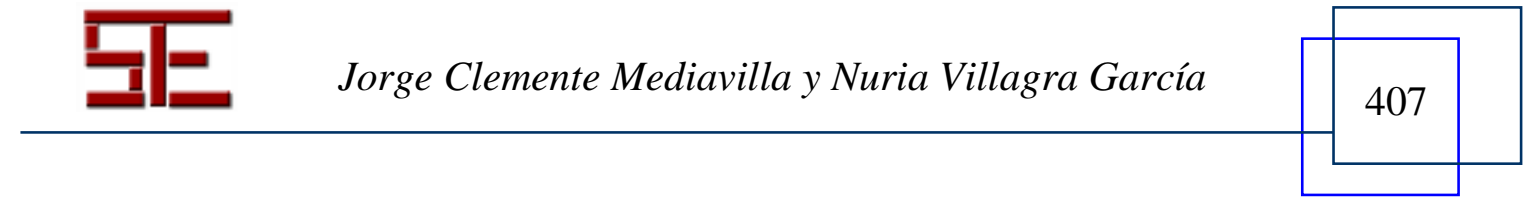




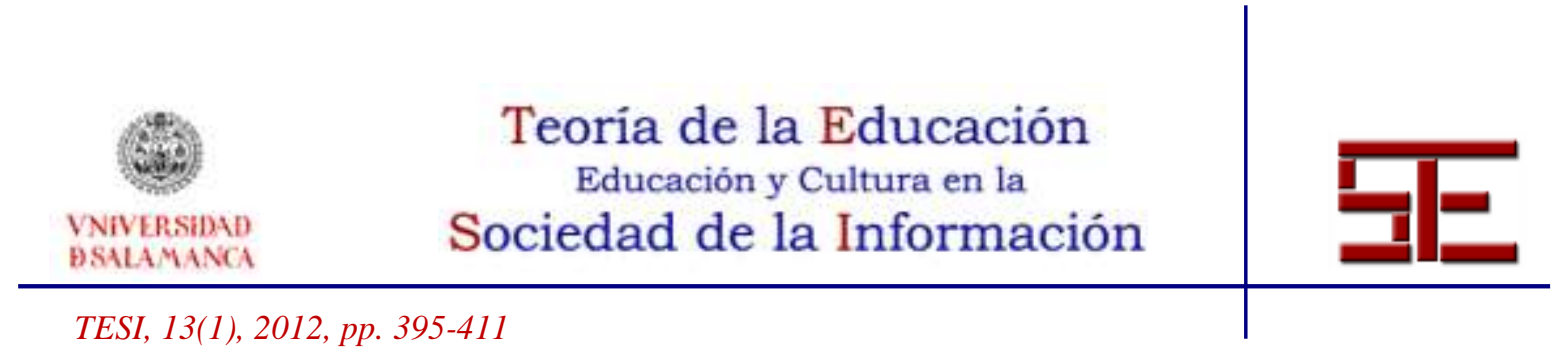

\section{3.- Resultados experimento en aula}

En las seis primeras preguntas se intentaba recoger información acerca de una serie de contenidos vertidos en la página web del cuestionario, tales como la facilidad de identificación del contenido a través del icono ( un 88,89\% respondió positivamente y un $11,11 \%$ contestó negativamente), la visualización de imágenes insertadas en dos documentos de texto (un 97,22 respondió que podía visualizarlo en ambos casos), la sencillez en la navegación de un documento HTML (ningún alumno adujo problemas), el funcionamiento correcto de un vínculo (un 94,44\% respondió afirmativamente), la visualización correcta de documentos PDF (un 66,67\% contestó positivamente), el tiempo transcurrido hasta el inicio de la reproducción de un archivo de vídeo (un $77,78 \%$ eligió la opción que implicaba una menor duración).

En la séptima pregunta del cuestionario, se requería a los alumnos que indicaran el navegador que utilizaban habitualmente cuando accedían al Campus Virtual. De un total de ocho navegadores listados (Internet Explorer, Opera, Mozilla Firefox, Netscape Navigator, Safari, Camino, Google Chrome, Otro), sólo fueron marcados cuatro de ellos. Nuevamente, los dos más utilizados fueron Mozilla Firefox (50\%) e Internet Explorer $(36,11 \%)$.

Con las dos últimas preguntas se pretendía recoger más información de los aspectos técnicos negativos de ambas plataformas, al preguntarse qué errores se detectaban en la utilización en el Campus Virtual de archivos de texto, de audio y vídeo, PDF, presentaciones, HTML e hipervínculos.

En ambos casos -Moodle y WebCT-, un porcentaje muy similar (94\%) de los alumnos no detectó errores en los Archivos de Texto. En cuanto al manejo de archivos PDF ocurría algo similar, con un 91,43\% de los alumnos que no halló fallos en Moodle, y un 97,22\% en el caso de WebCT. Respecto a la utilización de archivos PPT y PPS, en Moodle la totalidad de quienes respondieron el cuestionario refirió no haber encontrado errores, algo muy similar a lo que contestaron en WebCT (94,29\%); y en cuanto a los archivos de vídeo, en Moodle un 90,91\% no encontró errores y en WebCT fue la totalidad del alumnado. Respecto a los archivos de audio, en ambas plataformas todos los alumnos indicaron no tener problema alguno, al igual que en la utilización de los Documentos HTML. Finalmente, se obtuvieron resultados idénticos en la no detección de errores en los Hipervínculos (88\%) mientras que el $11 \%$ señaló su disfunción entre las posibilidades de error.

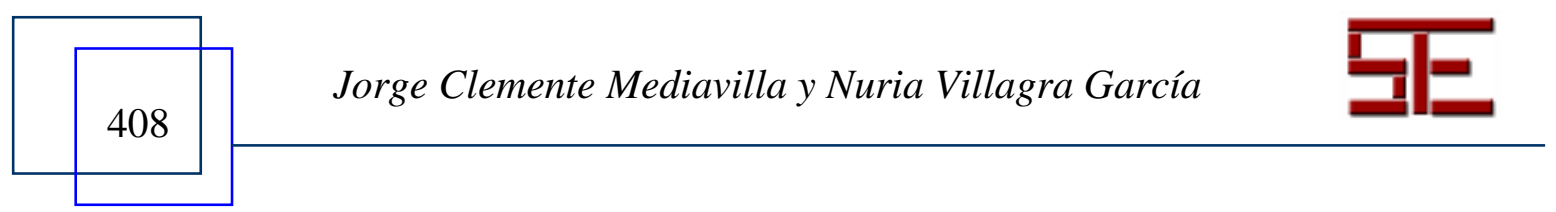




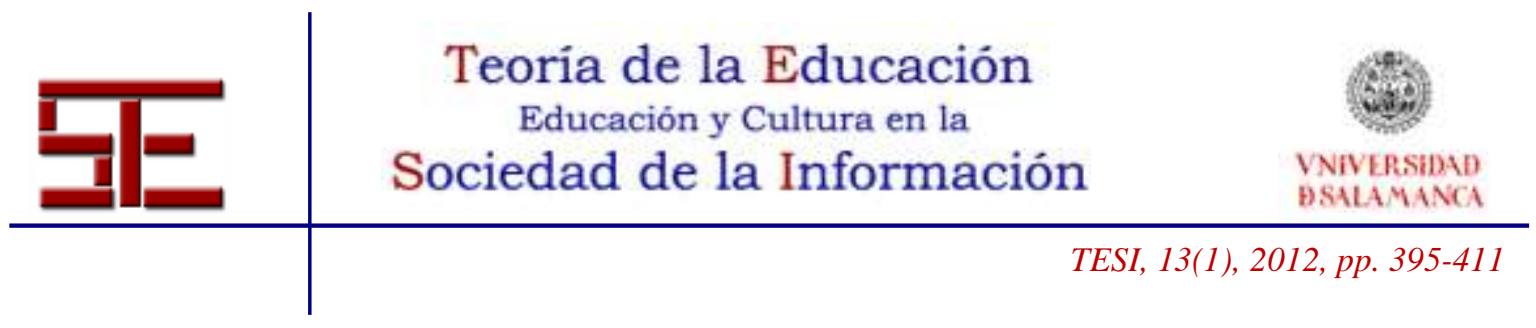

\section{4.- DISCUSIÓN}

Tras el análisis de los resultados obtenidos a través de la metodología planteada, podemos destacar una serie de argumentos que posibiliten la comprensión de la situación actual en lo referente a la utilización de plataformas de enseñanza virtual en el caso que nos ha ocupado.

Los resultados del experimento del profesorado ponen de manifiesto que aunque ambas plataformas tienen funcionalidades similares, la usabilidad, la accesibilidad y las posibilidades de comunicación son más dinámicas en Moodle. Este resultado contrasta con la valoración que los alumnos realizan de ambos espacios virtuales, donde WebCT destaca notablemente sobre Moodle. Esta última afirmación, fruto de los resultados de la investigación, obedece a que WebCT lleva implantada muchos años en la Universidad Complutense y ha sido utilizada habitualmente por el profesorado para la impartición de sus asignaturas, mientras que Moodle es mucho más reciente. En consecuencia, los alumnos están acostumbrados a utilizar en mayor medida la plataforma WebCT y ello hace que obtenga puntuaciones más elevadas.

Por otro lado, es destacable que ambas plataformas están infrautilizadas, tanto por parte del profesorado, en primera instancia, como del alumnado; las dos partes implicadas deberían hacer un uso más amplio de este espacio, aprovechando las posibilidades que ofrecen las herramientas virtuales. En lo referente al profesorado, las herramientas más empleadas son las relacionadas con el volcado de contenidos en formato texto y el uso del correo electrónico, no utilizándose adecuadamente el resto de funcionalidades existentes. Así, la utilización del espacio virtual por parte del docente en ambas plataformas es valorada como un uso estático, como un "almacén de contenidos", de ahí que los discentes visiten poco el espacio virtual y, cuando lo hacen, su tiempo de estancia sea breve y se centre principalmente en la descarga de los documentos anteriormente comentados. En definitiva, los alumnos demandan un papel más activo y participativo en el Campus Virtual, tal y como queda de manifiesto cuando se les preguntó por la posibilidad de un espacio para subir y compartir archivos con los compañeros, con una mayoría de respuestas favorables.

Este uso poco dinámico y participativo del espacio virtual también se observa cuando se analizan los datos que se refieren al empleo de las herramientas de comunicación, que permiten interactuar a alumnos y profesores. Este diálogo debería potenciarse, haciendo partícipes a ambas partes de todo lo relacionado con la asignatura y quizá más allá,

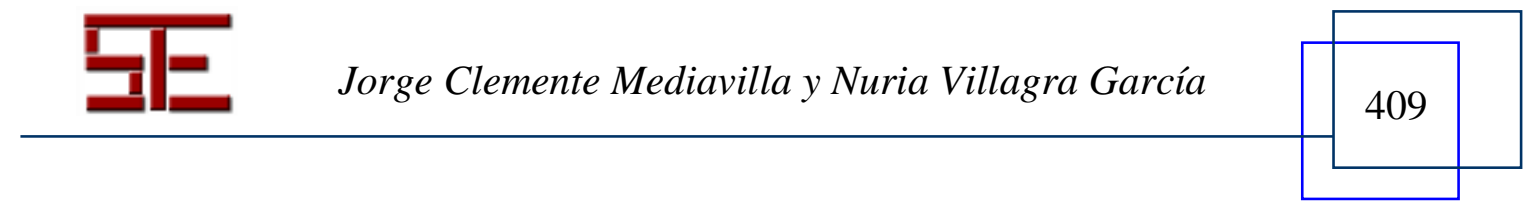




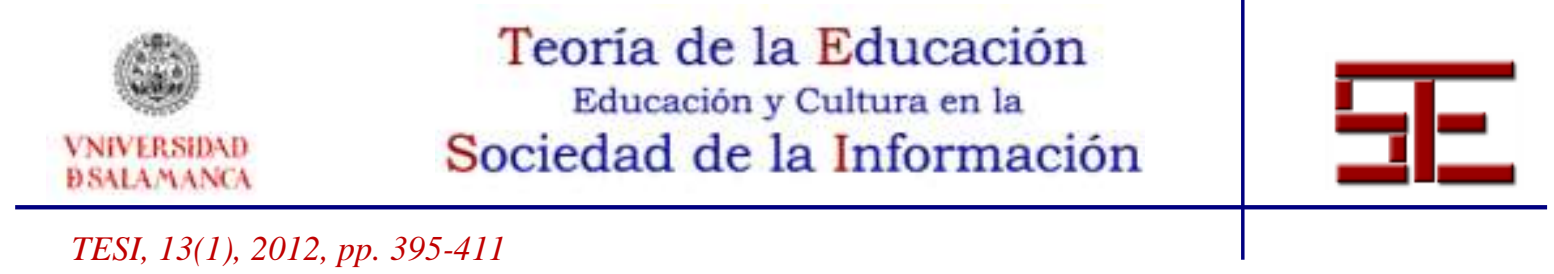

asemejándose a lo que podría ser una mini-red social, algo a lo que están acostumbrados los jóvenes universitarios de nuestro país. Los alumnos dedican mucho tiempo a Internet, pero sólo una pequeña parte es destinado al Campus Virtual UCM, porque para comunicarse y relacionarse utilizan otros espacios, tales como las redes sociales (en España Tuenti y Facebook). En consecuencia, sería interesante saber si el Campus Virtual puede potenciar más las herramientas de comunicación y participación, o si, por el contrario, debería complementarse con las redes sociales anteriormente citadas.

\section{5.- BIBLIOGRAFÍA}

Area, M. (2001). Las redes de ordenadores en la enseñanza universitaria: Hacia los campus virtuales. En García-Valcárcel, A. Didáctica universitaria (pp. 231-260). Madrid: La Muralla.

Belanger, F. \& Jordan, D. H. (2000). Evaluation and implementation of distance learning: technologies, tools and techniques. Hershey, PA: Idea Group Publishing.

Cabero, J. (2002). Las TICs en la Universidad. Sevilla: Editorial MAD.

Campbell, D. \& Stanley, J. (1995). Diseños experimentales y cuasi experimentales en la investigación social. Buenos Aires: Amorrortu.

Clares, J. (2000). Telemática, enseñanza y ambientes virtuales colaborativos. Comunicar, 14, 191-199.

Cortina-Pérez, B. (2008). Teaching and learning English through WebCT Tools: Promoting digital scaffolding. The International Journal of Technology Knowledge and Society, 2 (5), 129-138.

Garrison, D. R. \& Anderson, T. (2005). El e-learning en el siglo XXI: Investigación y práctica. Barcelona: Octaedro.

Llorente, M. C. (2007). Moodle como entorno virtual de formación al alcance de todos. Comunicar, 28, 197-202.

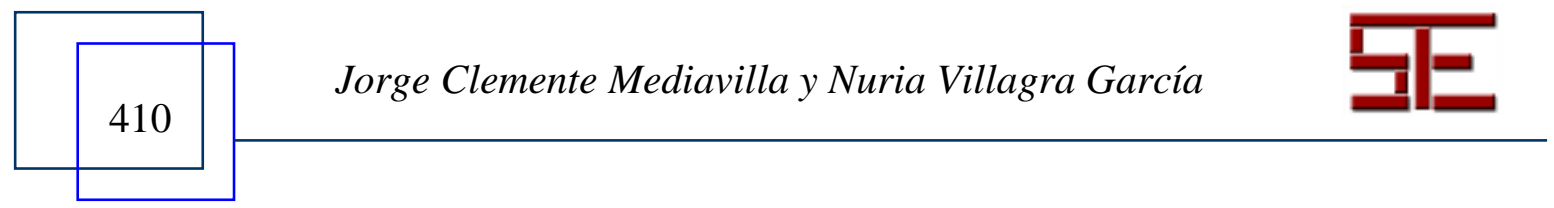




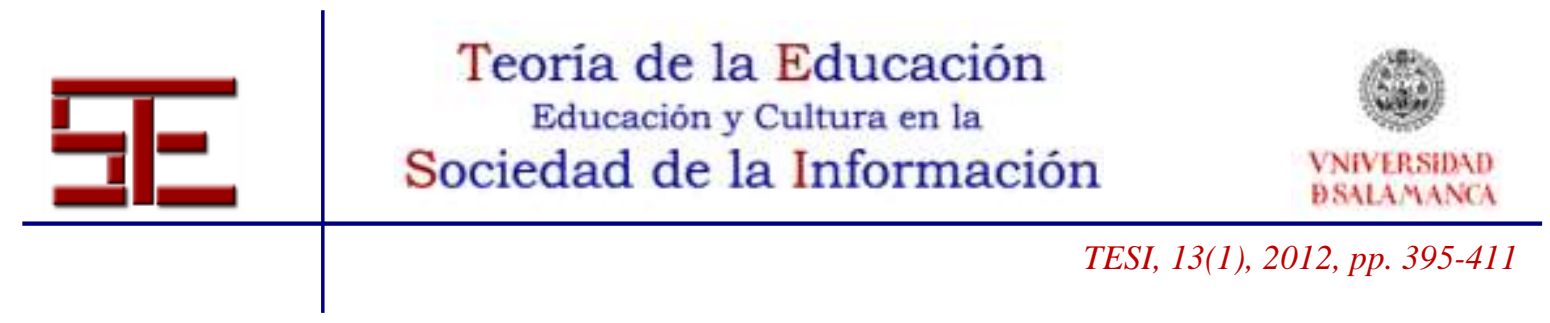

Novitzki, J. (2000). Asynchronous learning tools: what is really needed, wanted and used? En Aggarwal, A.K. (Ed.), Web-based learning and teaching technologies: opportunities and challenges (pp. 60-78). Hershey, PA: Idea Group Publishing.

Pérez, M. (2008a). Campus virtuales en universidades presenciales: ¿sueñan los estudiantes con profesores eléctricos? Revista Latinoamericana de Tecnología Educativa, 7 (1), 85-95.

Pérez, M. (2008b). Universidades presenciales y entornos virtuales de aprendizaje: una aproximación a la perspectiva del profesorado ante el e-learning. Revista Electrónica Teoría de la Educación. Educación y Cultura en la Sociedad de la Información, vol. 9 (1) febrero, 183-196.

Salmerón, H., Rodríguez, S. \& Gutiérrez, C. (2010). Metodologías que optimizan la comunicación en entornos de aprendizaje virtual. Comunicar, 34, 163-171.

Van Dusen, G. C. (1997). The Virtual Campus: Technology and reform in higher education. ASHE-ERIC Higher Education Report, vol. 25, n.5. Washington, DF: The George Washington University, Graduate School of Education and Human Development.

Para citar el presente artículo puede utilizar la siguiente referencia:

Clemente Mediavilla, J. y Villagra García, N. (2012). Nuevos desarrollos en el campus virtual UCM: estudio exploratorio sobre las plataformas e-learning en los estudios de comunicación audiovisual y publicidad. Revista Teoría de la Educación: Educación y Cultura en la Sociedad de la Información. 13(1), 395-411 [Fecha de consulta: $\mathrm{dd} / \mathrm{mm} / \mathrm{aaaa}]$.

http://campus.usal.es/ revistas_trabajo/index.php/revistatesi/article/view/8813/9021

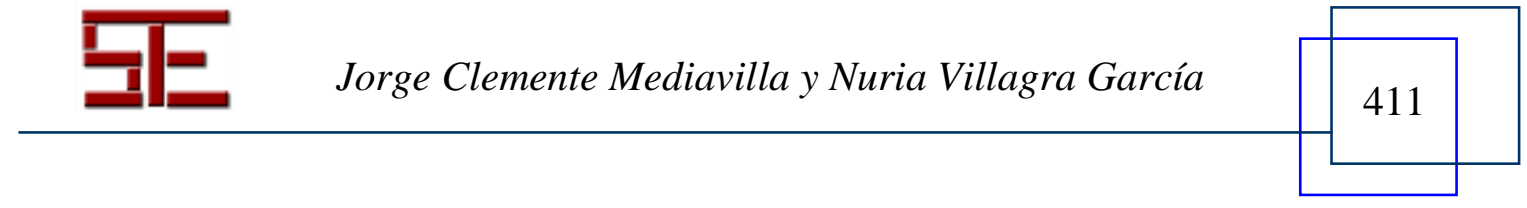

